\title{
Breast Cancer Synchronism With Lung Cryptococcosis: A Case Report
}

\author{
Chun-Hung Lin ${ }^{\mathrm{a}}$, Chih-En Tseng, ${ }^{\mathrm{b}, \mathrm{e}}$, Shu-Mei Chang ${ }^{\mathrm{b}}$, Moon-Sing Lee ${ }^{\mathrm{c}, \mathrm{e}}$, Hon-Yi Lin $^{\mathrm{c}, \mathrm{e}}$, \\ Yu-Chieh Su ${ }^{\mathrm{d}, \mathrm{e}}$, Wen-Yen Chiou ${ }^{\mathrm{c}, \mathrm{e}}$, Shih-Kai Hung ${ }^{\mathrm{c}, \mathrm{e}, \mathrm{f}}$
}

\begin{abstract}
Pulmonary metastasis is common among patients with breast cancer, and 18-fluoro-2-deoxyglucose positron emission tomography (FDG-PET) is a useful diagnostic tool for identifying breast cancer with metastatic lesions. However, inflammation or infection can often interfere with diagnosis. We report on a 53-year-old female whose diagnosis included malignant breast cancer concurrent with lung cryptococcosis. A PET retention index greater than $10 \%$ has been used as a cutoff for malignancy; however, the retention index is not useful for differentiation if the initial standard uptake value is low. As such, a pathologic diagnosis should be considered.
\end{abstract}

Keywords: Breast cancer; Cryptococcosis; Positron emission tomography

\section{Introduction}

Pulmonary metastasis is common among patients with breast cancer, especially in those at an advanced stage [1]. Chest computed tomography (CT) is the standard imaging modality used to evaluate pulmonary nodules [2]. With the development of modern techniques, 18-fluoro-2-deoxyglucose positron emission tomography (FDG-PET) has become a useful

Manuscript accepted for publication July 18, 2013

${ }^{\text {a}}$ Department of General Surgery, Buddhist Dalin Tzu Chi General Hospital, Chiayi, Taiwan

${ }^{\mathrm{b}}$ Department of Anatomic Pathology, Buddhist Dalin Tzu Chi General Hospital, Chiayi, Taiwan

${ }^{\mathrm{c}}$ Department of Radiation Oncology, Buddhist Dalin Tzu Chi General Hospital, Chiayi, Taiwan

${ }^{\mathrm{d} D e p a r t m e n t ~ o f ~ H e m a t o l o g i c a l ~ O n c o l o g y, ~ B u d d h i s t ~ D a l i n ~ T z u ~ C h i ~}$ General Hospital, Chiayi, Taiwan

'School of Medicine, Tzu Chi University, Hualian, 9706, Taiwan ${ }^{\mathrm{f}}$ Corresponding author: Shih-Kai Hung, Department of Radiation Oncology, Buddhist Dalin Tzu Chi General Hospital, 2, Ming Sheng Road, Dalin, Chiayi, Taiwan. Email: oncology158@yahoo.com.tw

doi: http://dx.doi.org/10.4021/jmc1406w tool for identifying breast cancer with metastatic lesions [3]. However, inflammation or infectious diseases can often interfere with the diagnosis [4]. In this report, we document a case of breast cancer concurrent with lung cryptococcosis and review the related literature.

\section{Case Report}

A 53-year-old female patient was shown to have malignant breast cancer, as determined by excision biopsy. The patient was arranged to have a whole-body survey before radial surgery. Unfortunately, a lung mass was noted by CT. The patient then underwent PET/CT for further differential diagnosis. Following intravenous injection of $10 \mathrm{mCi} \mathrm{F-18} \mathrm{FDG}$ and a standard uptake period, the patient was imaged on a PET scanner and the images were reconstructed. PET/CT revealed a right breast nodule in accordance with pathologyapproved malignancy (Fig. 1). In addition, PET/CT also revealed a nodule $(19 \mathrm{~mm})$ over the right lower lung (Fig. 2). The initial standard uptake value (SUV) of the right lower lung nodule was 1.7 , the delay SUV was 2.5 , and the reten-

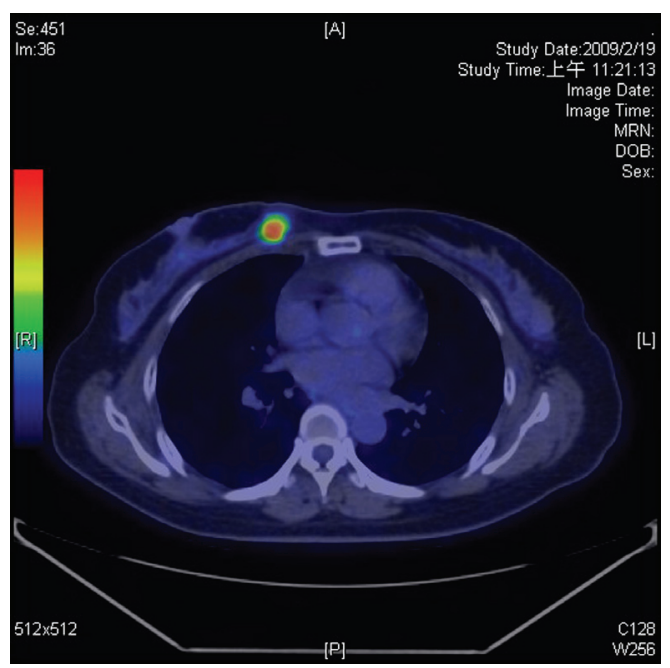

Figure 1. PET/CT revealed a right breast nodule in accordance with pathology-approved malignancy. 


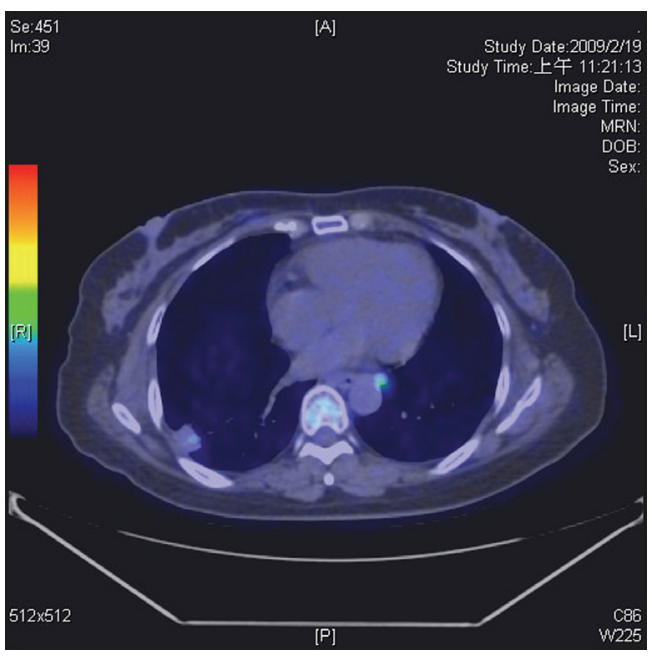

Figure 2. PET/CT revealed a nodule over the right lower lung.

tion index was $41 \%$. Because the lung mass was solitary, we performed a partial mastectomy plus sentinel node for the breast lesion and wedge resection for the lung lesion after combined meeting. The pathology of the breast lesion indicated infiltrating ductal carcinoma, stage pT1cN1micM0. In addition, pathology of the lung lesion revealed positive staining for PAS, PASD, and mucicarmine and negative for acid fast staining. The sections showed lung tissue with a large caseating granuloma with abundant fungal elements. The morphology was that of cryptococcosis (Fig. 3). After final approved, the patient received sequent antibiotic therapy and adjuvant cancer therapy without incidence.

\section{Discussion}

Breast cancer is considered a systemic disease, and lung metastasis is common in these patients [1]. However, a series of studies have reported $5-22 \%$ of lung nodules are shown to be non-malignant following pathological confirmation [4-7]. Tanaka et al. evaluated 52 patients with breast cancer who underwent surgery for pulmonary nodules. The incidence of metastatic lesions, primary lung cancer, and non-malignancy was $61 \%, 17 \%$, and $22 \%$, respectively, for single lung nodules [4]. Because a single pulmonary nodule is not always a result of pulmonary metastasis, surgery is one option for obtaining pathologic confirmation.

An immunocompromised status caused by chemotherapy or tumor involvement may contribute to infection with malignancy. Cryptococcus infections have been reported in many patients with cancer, including those with nasopharyngeal carcinoma, leukemia, breast cancer, and lung cancer [710]. In addition, healthy persons with a history of exposure to pigeons have a higher rate of infection [11]. This is also true for patients that live in tropical climates and those who

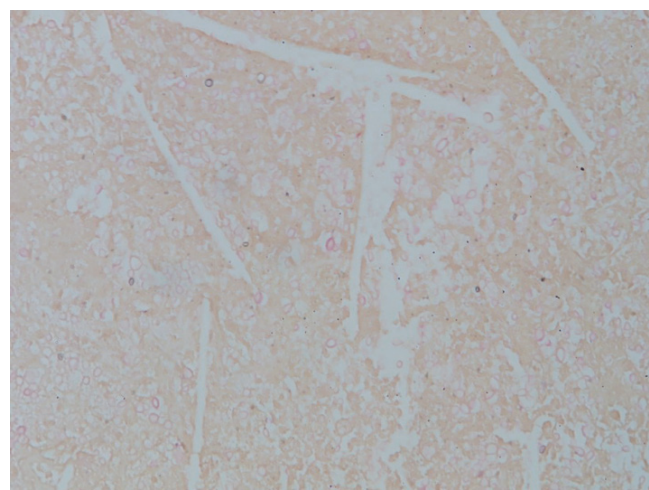

Figure 3. Pathology of the lung lesion revealed positive staining for mucicarmine.

come in contact with the eucalyptus trees, with evidence indicating a significant prevalence in Taiwan [12]. Together, these factors may contribute substantially to the occurrence of cryptococcus infections.

Because pulmonary nodules mimic pulmonary metastases, obtaining an accurate diagnosis is important. PET is a useful and non-invasive tool for determining metastatic lesions, especially in lung, breast, and esophageal cancers [13]. Xiu et al. demonstrated that dual-time PET imaging can improve accuracy in the evaluation of lung nodules. A retention index greater than $10 \%$ has been used as a cutoff for determining malignancy [14]. However, Chen et al. showed that the retention index may not be useful for discrimination, especially if the initial standard uptake value is less than 2.5 [15]. Although the retention index was $41 \%$ in our case, the initial value of SUV $\max _{\text {max }}$ was only 1.7. Thus, PET was not suitable for differentiating between malignant and benign disease in this patient.

Breast cancer with a single pulmonary nodule can be metastatic, primary, or benign and PET analysis can be used to differentiate between these states. However, PET is not useful for differentiation if the initial standard uptake value is low. In these cases, a pathologic diagnosis should be considered.

\section{Conflict of Interest}

The authors declare no conflicts of interest.

\section{References}

1. Rashid OM, Takabe K. The evolution of the role of surgery in the management of breast cancer lung metastasis. J Thorac Dis. 2012;4(4):420-424.

2. Detterbeck FC, Grodzki T, Gleeson F, Robert JH. Imaging requirements in the practice of pulmonary metasta- 
sectomy. J Thorac Oncol. 2010;5(6 Suppl 2):S134-139.

3. Brennan ME, Houssami N. Evaluation of the evidence on staging imaging for detection of asymptomatic distant metastases in newly diagnosed breast cancer. Breast. 2012;21(2):112-123.

4. Tanaka F, Li M, Hanaoka N, Bando T, Fukuse T, Hasegawa S, Wada H. Surgery for pulmonary nodules in breast cancer patients. Ann Thorac Surg. 2005;79(5):17111714; discussion 1714-1715.

5. Chang EY, Johnson W, Karamlou K, Khaki A, Komanapalli C, Walts D, Mahin D, et al. The evaluation and treatment implications of isolated pulmonary nodules in patients with a recent history of breast cancer. Am J Surg. 2006;191(5):641-645.

6. Casey JJ, Stempel BG, Scanlon EF, Fry WA. The solitary pulmonary nodule in the patient with breast cancer. Surgery. 1984;96(4):801-805.

7. Ou KW, Hsu KF, Cheng YL, Hsu GC, Hsu HM, Yu JC. Asymptomatic pulmonary nodules in a patient with early-stage breast cancer: Cryptococcus infection. Int $\mathrm{J}$ Infect Dis. 2010;14(1):e77-80.

8. Sun LM, Chen TY, Chen WJ, Hsieh MJ, Liu JW, Huang CC, Wang CJ. Cryptococcus infection in a patient with nasopharyngeal carcinoma: imaging findings mimicking pulmonary metastases. Br J Radiol. 2002;75(891):275278.

9. Wells GM, Gajjar A, Pearson TA, Hale KL, Shenep JL. Brief report. Pulmonary cryptosporidiosis and Crypto- coccus albidus fungemia in a child with acute lymphocytic leukemia. Med Pediatr Oncol. 1998;31(6):544546.

10. Kimura M, Kaufman L, Maekura S, Teramura K, Satou T, Hashimoto S. Pulmonary cryptococcosis due to a capsule-deficient strain confused with metastatic lung cancer. Mycopathologia. 1997;140(2):65-68.

11. Newberry WM, Jr., Walter JE, Chandler JW, Jr., Tosh FE. Epidemiologic study of Cryptococcus neoformans. Ann Intern Med. 1967;67(4):724-732.

12. Chen YC, Chang SC, Shih CC, Hung CC, Luhbd KT, Pan YS, Hsieh WC. Clinical features and in vitro susceptibilities of two varieties of Cryptococcus neoformans in Taiwan. Diagn Microbiol Infect Dis. 2000;36(3):175183.

13. Visioni A, Kim J. Positron emission tomography for benign and malignant disease. Surg Clin North Am. 2011;91(1):249-266.

14. Xiu Y, Bhutani C, Dhurairaj T, Yu JQ, Dadparvar S, Reddy S, Kumar R, et al. Dual-time point FDG PET imaging in the evaluation of pulmonary nodules with minimally increased metabolic activity. Clin Nucl Med. 2007;32(2):101-105.

15. Chen CJ, Lee BF, Yao WJ, Cheng L, Wu PS, Chu CL, Chiu NT. Dual-phase 18F-FDG PET in the diagnosis of pulmonary nodules with an initial standard uptake value less than 2.5. AJR Am J Roentgenol. 2008;191(2):475479. 